\title{
The North Texas Advanced Research and Technology Institute: Instrumentation for Materials Characterization and Education
}

\author{
Brian P. Gorman and Michael J. Kaufman
}

Department of Materials Science and Engineering, University of North Texas, Denton, TX, 76203

The University of North Texas' Department of Materials Science and Engineering has been undergoing a large scale expansion in the past couple of years, including the acquisition of a $550,000 \mathrm{ft}^{2}$ research park (a former Texas Instruments facility) and the addition of several faculty and research personnel positions. A major part of this expansion has included the simultaneous formation of the North Texas Advanced Research and Technology Institute (ARTI) along with several major equipment acquisitions. ARTI is currently being initiated as a multi-user facility with many collaborative research opportunities.

Equipment available in ARTI includes several state of the art instruments such as: Field emission HRTEM with STEM, EDS, PEELS, EFTEM, HAADF, cold and hot stages, tomography stages, and in-situ electrical probing; Dual column field emission SEM / FIB with EDS, EBSD, nanomanipulator with mechanical and electrical characterization, and STEM and TL detection; Local electrode atom probe (LEAP) with voltage pulsing for atomic scale tomographic characterization; High resolution XRD with a 2-D detector, thin film geometries, SAXS, in-plane diffraction, pole figure analyses, and high temperature capabilities up to $1500^{\circ} \mathrm{C}$; Environmental SEM equipped with STEM, Peltier stage, $1500^{\circ} \mathrm{C}$ hot stage, EDS, and EBSD; Teaching grade SEMs, TEMs, and XRDs equipped with digital imaging, EDS, and heating and straining stages; Atomic force / scanning tunneling microscopy with magnetic and lateral force microscopy capabilities; a complete optical microscopy laboratory with digital imaging in the visible and IR; Mechanical properties characterization including nanoindentation with high load, continuous stiffness, and AFM modules as well as various tribometers and impact testers; and finally materials science and biological sample preparation facilities.

The combination of these instruments gives ARTI a unique opportunity for characterization. Especially interesting to many existing faculty is the ability to characterize 1 to 3-dimensional structures at length scales from mm to sub-nm. Current research projects include polymer / inorganic nanocomposites, microelectronic devices such as strained semiconductors and high- and low-k dielectrics, metallurgical thin films, solid oxide fuel cells, optoelectronic materials for displays and photovoltaics, biomaterials, and structural ceramics.

Laboratory and classroom courses dealing with the instrumentation are taught in the UNT MSE department every semester. Classes are taught at the UNT research park as well as online. TEM courses taught in the classroom offer students the advantages of real time TEM observation and use through remote operation. All laboratory courses offer students hands-on time with each instrument, which ultimately results in the students being able to use the instruments independently of the instructors.

Continued expansion and collaborations are expected and encouraged within ARTI. Users interested in ARTI facilities are encouraged to contact the authors. 